\title{
Difficulties in recognizing granulomatosis with polyangiitis (GPA) in elderly patients undergoing diagnostic thoracotomy twice - a report of two cases
}

\begin{abstract}
Granulomatosis with polyangiitis (GPA) is defined as a necrotizing granulomatous inflammation usually involving the upper and lower respiratory tract with necrotizing vasculitis affecting predominantly small to medium vessels. Because of non-specific symptoms, its radiological presentation, and the diversity of its clinical expression, it is not uncommon for it to be misdiagnosed, especially in the elderly. Although biopsy and histological examination seem to be essential for GPA diagnosis, their results are sometimes ambiguous and not helpful in making a decision. In this report, we present difficulties in the recognition of GPA in two elderly patients in whom, despite twice performing a diagnostic thoracotomy, GPA was recognized almost 4 and 6 years after the first symptoms.
\end{abstract}

Key words: GPA, elderly patients, diagnostic difficulties

Adv Respir Med. 2019; 87: 258-264

\section{Introduction}

Granulomatosis with polyangiitis (GPA) is an uncommon immunologically-mediated systemic small-vessel vasculitis that is characterized by necrotizing granulomatous inflammation usually involving the upper and lower respiratory tract. There is also necrotizing vasculitis affecting predominantly small to medium vessels frequently coexisting with necrotizing glomerulonephritis [1]. It can occur at any age, although it is primarily a disease of older adults, with the peak age between 65 and 74 years old [2]. Because the upper respiratory tract is involved in $70-100 \%$ cases, classic otorhinolaryngologic symptoms - ear, nose and throat (ENT) manifestations - may be the first clinical presentation of the disease $[3,4]$. Some patients may progress to a more generalized form of disease, usually involving the lungs, skin and/or kidneys, with pauci-immune necrotizing crescentic glomerulonephritis [5]. Classically, the acronym ELK is used to describe the common organ involvement in GPA (E - ENT, L - lungs, K - kidneys) [4].

According to the BSR (British Society for Rheumatology) and the BHPR (British Health Professionals in Rheumatology) guidelines, the following criteria must be fulfilled prior to a diagnosis of antineutrophil-cytoplasmic antibodies (ANCA)-associated vasculitis (AAV): 1) Symptoms and signs characteristic of systemic vasculitis; 2) At least one of the following: a) histological evidence of vasculitis and/or granuloma formation; b) positive serology for ANCA; c) specific indirect evidence of vasculitis; 3 ) No other diagnosis to account for symptoms or signs [6]. In practice, the diagnosis of GPA is usually based on clinical features and the presence of ANCA and is supported, whenever possible, by histology.

Early diagnosis and treatment of GPA is important but, unfortunately, determining the correct diagnosis may cause difficulties, especially in the elderly. Systemic features (such as

Address for correspondence: Justyna Fijołek, Third Department of Pneumonology, National Tuberculosis and Lung Diseases Research Institute, Warsaw, Poland; 
malaise, fevere and weight loss) can appear not only in AAV, but also in other diseases and may last from weeks to months before evidence of specific organ involvement [3]. On the other hand, somtimes clinical symptoms are scarce and even surgical biopsy with histologic examination is not decisive. The present paper describes difficulties in the recognition of GPA in two elderly women in whom, despite performing diagnostic thoracotomy twice, GPA was barely recognized 4 and 6 years after the first symptoms.

\section{Case report 1}

A 71 year old female presented the first symptoms in 2013. She complained of a moderate cough and general malaise. A chest radiograph showed bilateral multiple pulmonary nodules and consolidations which were confirmed by a chest computed tomography (CT) scan in 2014. Results of standard laboratory blood tests were normal. There was no haematuria nor proteinuria, but there was an increased level of anti-myeloperoxidase (MPO-ANCA) to $95 \mathrm{Ru} / \mathrm{mL}$ (relative units per milliliter, $N \leq 20$ ) and an increased titer of the anti-nuclear antibodies (ANA) with the presence of double-stranded deoxyribonucleic acid (anti-dsDNA) antibodies and lupus anticoagulant (LA) proteins. During bronchoscopy, no pathology was found. In June 2014, the patient underwent diagnostic left thoracotomy with a wedge-like excision of the one nodule. In the resected specimen, inflammation composed of lymphocytes, plasma cells and neutrophils with focal interstitial fibrosis was described. Because the patient had mild symptoms and pulmonary function tests were normal, no treatment was administered.

In August 2015, the control CT showed a partial spontaneous regression of pulmonary lesions. However, in February 2016, clinical symptoms intensified. The patient now experienced a productive cough, exercise dyspnoea and general malaise. The CT revealed the progression in the lungs: the persistent nodules had been enlarged and new nodules and ground-glass opacities appeared. The CT was unambiguous and the pathology was still estimated to be interstitial lung disease. It is especially interesting to note that based on previous serological findings, systemic lupus erythematosus (SLE) was suspected. Because cancer was also considered, a transthoracic lung biopsy guided by CT was performed, but it showed only lymphocytes and neutrophils without neoplastic cells. A bronchoscopy was performed and no pathology was found. Results of mycobac- terial and fungal cultures were negative. In March 2016, the patient underwent a second diagnostic thoracotomy (this time, of the right side) with the wedge-like excision of the part of the middle and lower lobes. In the resected specimen, intensive fibrosis with chronic inflammation composed of giant cells and neutrophils was described.

The patient was admitted to our Institute for the first time two months after the second thoracotomy. She was slightly weak and had a weak cough, but control CT again showed a spontaneous partial regression of the pulmonary lesions. The histological revision of both of the resected specimens (taken in 2014 and 2016) revealed inflammation with granulomas containing giant cells, components of vasculitis, small focuses of alveolar hameorrhage, and organizing pneumonia. In conclusion, the pathology study suggested GPA as well as lesions in the course of connective tissue disease, especially SLE. The patient did not present with any other organ involvement except for the lungs and had no symptoms of lupus or other connective tissue disease. Laboratory tests (neutrophil counts, CRP, urine sediment) remained normal and furthermore, the initially positive ANCA and ANA tests had become negative. She was left under observation without treatment.

After nearly one year, in January 2017, the patient was admitted to our Institute again. She presented with a 3-week history of an increasingly productive cough with blood-tinged sputum and increasing malaise. Additionally, she now also presented with nasal symptoms. Laboratory investigation showed an elevated white blood cell and neutrophil count, an increase in the CRP level, proteinuria and haematuria with glomerular filtration rate (GFR) impairment. The CT demonstrated progression of the nodules in the lungs (Figure 1). Additionally, a head CT revealed sinusistis with bone destruction. Serological testing showed a slightly elevated level of MPO-ANCA to $29 \mathrm{Ru} / \mathrm{mL}$ with a slightly positive ANA test. Based on the clinical course (lung, sinus, kidney involvement), histological examination and presence of ANCA, a diagnosis of GPA (MPO-ANCA AAV) was established. The patient was treated with prednisone and cyclophosphamide and responded well. Follow-up CT 3 months after treatment showed resolving pulmonary lesions and complete remission after 2 years.

\section{Case report 2}

The disease of a 72-year old female also started in 2013 with abnormal laboratory test results 

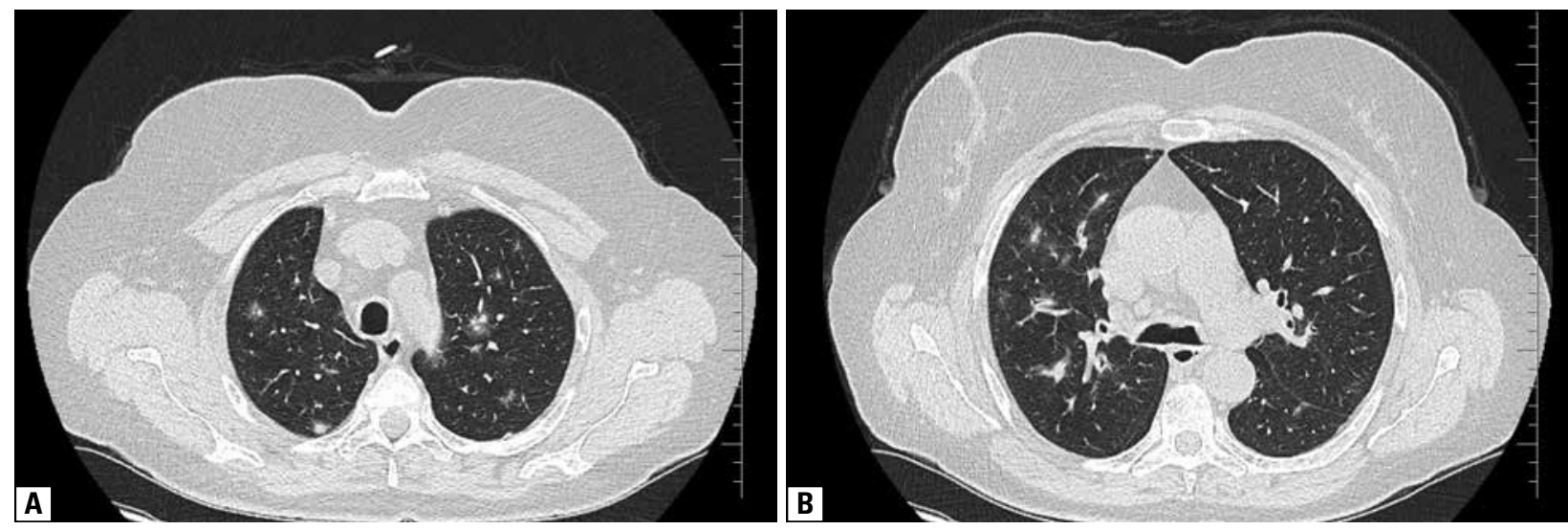

Figure 1 A, B. Chest CT (2017), lung windows, tranverse sections: the study shows progression of pulmonary lesions: more numerous small nodules and small ground-glass opacities in both upper lobes

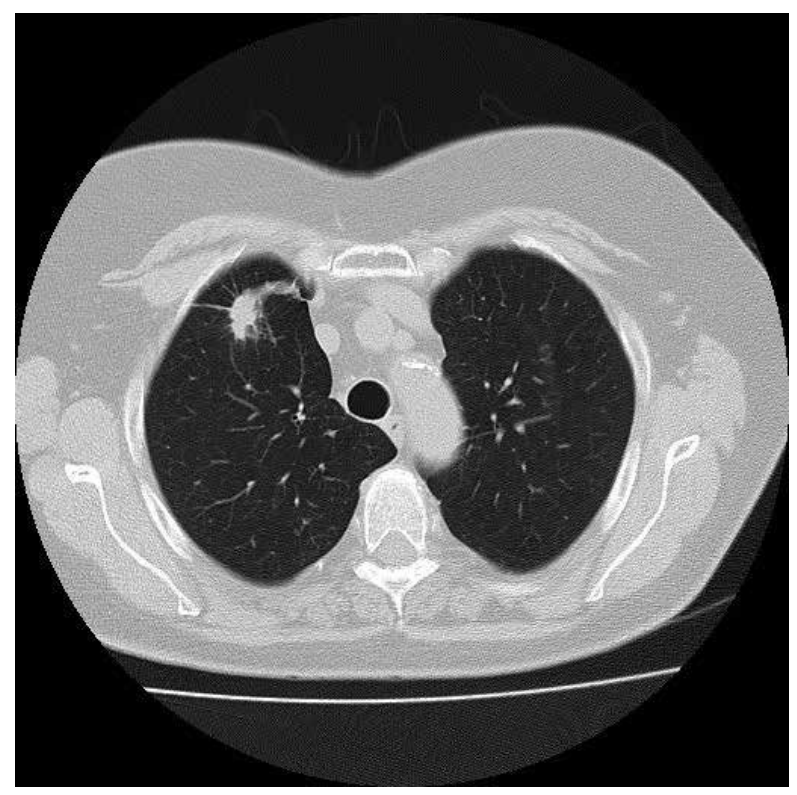

Figure 2. Chest CT (2013), lung window, trnsverse section: a solitary spiculated nodule in the right upper lobe

(a slight increase of the erythrocyte sedimentation rate-ESR). She did not have any clinical symptoms. Chest-X-ray showed a solid nodule $17 \times 20$ $\times 11 \mathrm{~mm}$ (millimeter) in the right upper lobe confirmed by CT (Figure 2). During bronchoscopy, no pathology was found. Lung cancer was suspected and, in November 2013, the patient underwent right diagnostic thoracotomy with a wedge-like excision of the nodule, but histologic examination revealed only inflammation and necrosis, with negative mycobacterial staining. Results of laboratory tests were normal and serological tests for ANCA and rheumatoid factor (RF) were negative. Because the patient had no clinical symptoms, it was decided to leave her under observation. Control CT performed after 6 months was normal.

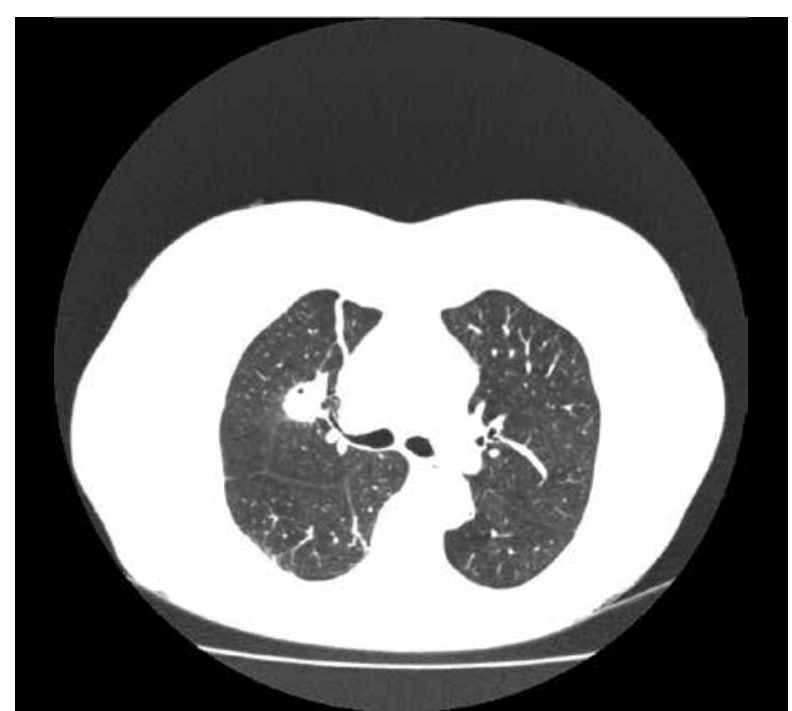

Figure 3. Chest CT (2015), lung window, transverse section: the sudy compared with the prior CT (Figure 2) shows a new solid nodule this time with the cavity - again in the right upper lobe, near a linear scar is visible associated with previous thoracotomy

However, in 2015, CT examination showed a new nodule within the cavity, again in the right lung (Figure 3). In April 2015, the patient underwent a second right diagnostic thoracotomy with a wedge-like excision of the nodule. This time, the resected specimen revealed chronic inflammation composed of lymphocytes with focal fibrosis but without necrosis, and without cancer cells or mycobacteria. During further follow-up visits, the patient remained asymptomatic and control CT scans repeated 3 and 18 months after were normal.

In 2017, an episode of haemoptysis occurred without other accompanying symptoms. The CT showed the appearance of two new cavitary nodules in the right lung (segment 2 and 8). During 
bronchoscopy, blood in the segmental bronchi was present, but results of mycobacterial and fungal cultures were again negative and revealed no malignancy. In histological examination of the bronchial slice, features of non-specific inflammation were found. Further, the fine needle aspiration biopsy was performed and showed inflammatory granulation without cancer cells. Results of laboratory tests and GFR remained normal, and ANCA were negative. During the further follow-up period haemoptysis did not recur, but the positron emission computed tomography (PET-CT) performed a few months later demonstrated an extension of the right lung nodular consolidations showing metabolic activity.

The patient was admitted to our Institute for the first time in January of 2018. She was in good performance status without any abnormalities during examination. Laboratory investigation showed a normal blood cell count with a normal count of neutrophils, without anemia, and with a slight increase in the level of CRP to $12 \mathrm{mg} /$ $\mathrm{dL}$ (miligram per deciliter, $\mathrm{N}<5$ ). There was no erythrocyturia or proteinuria and the GFR was not impaired. Because the CT showed further minor progression in the lung, the patient underwent bronchoscopy but no pathology was found, and mycobacterial and fungal cultures were negative. The possibility of GPA was considered, and diagnostics were extended to CT of the sinuses/ears, but laryngological examination was negative. Serological tests showed a slight non-specific increase in anti-proteinase 3 (PR3-ANCA) concentration $(22 \mathrm{Ru} / \mathrm{mL}, \mathrm{N} \leq 20)$. The histological revision of the resected 2013 and 2015 specimens revealed necrotizing granulomatous inflammation with histiocyte, neutrophil and lymphocyte infiltration centered on bronchi and bronchioles. The Grocott and periodic acid-Schiff (PAS) stains showed no fungal elements. The Ziehl-Neelsen stain was also negative. The pathology study suggested bronchocentric granulomtosis (BCG) requiring clinical differentiation with a less likely bronchocentric form of GPA. The patient had no obvious evidence of the underlying disease of BCG (serum IgE level was not elevated, there was no fungal hypersensitivity, and no features of either an allergic bronchopulmonary aspergillosis or mycobacteria) and she did not have other organ involvement typical for vasculitis. Because the patient was asymptomatic, and the diagnosis was uncertain, it was decided not to initiate steroid therapy. During further follow-up visits in March and July 2018, the patient was feeling good. CT control scans showed variable pulmonary nod- ules (spontaneous partial regression, after which nodules underwent a minor progression or intensification of cavity). ANCA tests were negative.

In January 2019, the patient was admitted to the Institute due to deterioration of their well-being. She presented with a 4-week history of productive cough with nasal symptoms and general malaise. Laboratory investigations showed elevated blood cell and neutrophil count with an increase in the level of CRP to $30 \mathrm{mg} / \mathrm{dL}$. Urine sediment and GFR were normal. The CT demonstrated progression of the cavitary nodules in the lungs (Figure 4). Additionally, sinusistis with bone destruction was found. In the serum ANCA, the concentration of PR3-ANCA increased to 42 $\mathrm{Ru} / \mathrm{mL}$. Based on the clinical course (sinus, lung involvement), histological examination and presence of ANCA, a diagnosis of GPA (AAV PR3-ANCA) was established. The patient was treated with prednisone and cyclophosphamide and responded well. Follow-up CT scan 3 months after treatment showed resolving pulmonary lesions.

\section{Discussion}

Diagnosis of GPA included specific symptoms, characteristic radiologic lesions and an appropriate microscopic pattern. Characteristic features for GPA are granulomatous inflammation of the upper and lower respiratory tracts in combination with necrotizing vasculitis affecting small vessels [1]. Typical symptoms included ENT manifestations [7]. Involvement of the lower respiratory tract was also common [8], and in over 30\% of cases was asymptomatic [9]. Interestingly, in $9 \%$ of patients, the lung was the only organ affected [9]. Radiographically, multiple and bilateral lung nodules occur in $40 \%$ to $70 \%$ of GPA cases, while cavitation occurs in $25 \%$ to $50 \%$ of these nodules [10]. The presence of ANCA, although not necessary, supports the diagnosis as it can be detected in up to $90 \%$ of patients with GPA [5]. To analyze our difficulty with diagnosing GPA, we are taking into account the following elements: the patients were older ( $>65$ years old), the first symptoms were connected only with the lungs, and the histological pattern did not contain the GPA features.

The accurate diagnosis of GPA can be difficult, especially in the elderly with disease initially limited to the lung like in our patients. The clinical presentation in older patients ( $>65$ years old) is similar to that seen in younger patients $(<65$ years old), although certain features may be more common [11]. In older patients 

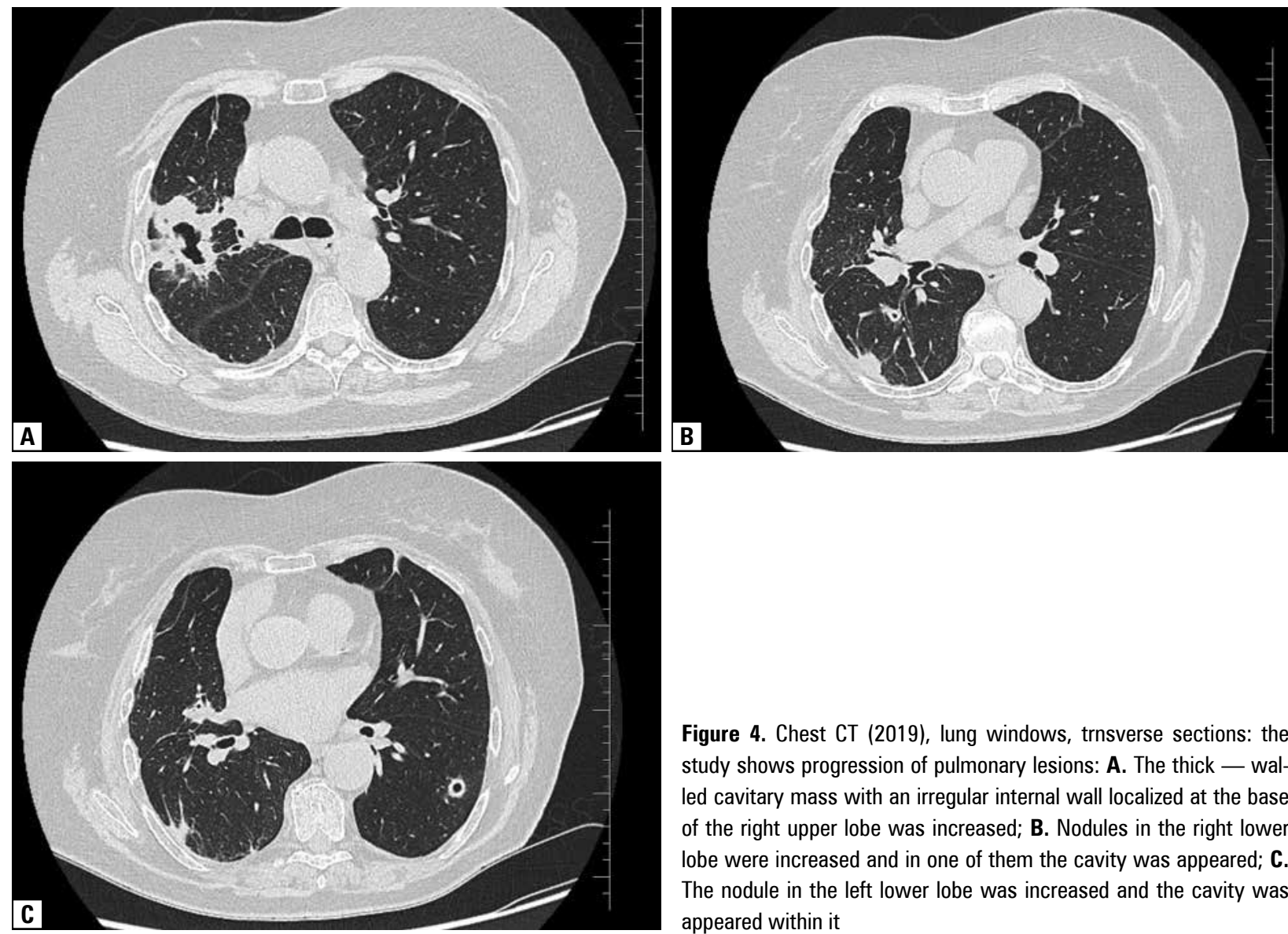

Figure 4. Chest CT (2019), lung windows, trnsverse sections: the study shows progression of pulmonary lesions: $\mathbf{A}$. The thick - walled cavitary mass with an irregular internal wall localized at the base of the right upper lobe was increased; B. Nodules in the right lower lobe were increased and in one of them the cavity was appeared; $\mathbf{C}$. The nodule in the left lower lobe was increased and the cavity was appeared within it

the upper respiratory tract is less commonly involved, although lung involvement is not uncommon and more frequently, MPO-ANCA are present [12-14]. In the elderly, nonspecific symptoms often occur, and diagnosis is often delayed $[2,15]$. Pulmonary nodules are a common radiological manifestation, but not specific for GPA, and the differential diagnosis includes infections caused by mycobacteria and fungi, metastatic solid tumors and other granulomatous processes [16]. It has been reported that pulmonary-limited GPA can simulate pulmonary adenocarcinoma [17]. There have also been cases masquerading as tuberculosis before the correct diagnosis was made [18]. In our patients, solitary lung nodules were the first symptoms of disease and, for a few years ( 4 and 6 , respectively), garnered the most attention in comparison with symptoms from other organs. Initially, metastatic cancer was also suspected, which has not been confirmed. Both patients underwent diagnostic thoracotomy twice and despite this, the appropriate primary diagnosis was not established.

GPA is also a challenge for the pathologist because of its varied histological pattern. In 1988, Mark et al. [19] reviewed open lung biopsies from
35 patients with GPA and established the relative diagnostic value of various histologic features. Authors concluded that the concept of GPA mainly as a vasculitis is too restrictive and does not include many cases with only extravascular histologic changes. Next, in 1990, Wegener himself wrote that „the vasculitis that accompanies the disease is a secondary feature that presents at a later stage and that this fact is often not heeded, and WG (Wegener's granulomatosis) is frequently mistaken for a systemic vasculitis" [20]. Evidence of how diverse the histological findings in GPA can be were provided by Travis et al. in 1991 [21]. They reported the pulmonary pathologic features in 87 open lung biopsies from 67 patients dividing their manifestations into major and minor. The major manifeastations included: 1) parenchymal necrosis with neutrophilic microabscesses and large or small areas of geographic necrosis (found in $84 \%$ of biopsy specimens); 2) vasculitis (different vascular changes identified in 94\%); and 3) granulomatous inflammation accompanied by an inflmammatory infiltrate composed of a mixture of neutrophils, lymphocytes, plasma cells, histiocytes, and eosinophils. Other features such as: 1) interstitial fibrosis (26\%); 2) alveolar 
hemorrhage (49\%); 3) tissue eosinophilia (100\%); 4) organizing intraluminal fibrosis (70\%); 5) endogenous lipoid pneumonia (59\%); 6) lymphoid aggregates (37\%); and 7) variety of bronchial/ bronchiolar lesions (51\%) were classified as a minor manifestation. Although minor pathologic lesions usually accompanied major lesions and were usually inconspicuous, in $18 \%$ of specimens a minor feature represented the dominant or major finding. In $12 \%$ of specimens, active vasculitis affecting veins or arteries could not be identified [21]. Thus, the spectrum of pathologic features that can occur in pulmonary GPA is broad and is characterized by a wide variation which results in a complicated differential diagnosis and difficulities in establishing an appropriate disease. This was excellently illustrated in our patients. Both underwent diagnostic thoracotomy twice and, despite the large lung specimens, histological examination was not conclusive and did not suggest another disease. In the first case, non-specific inflammation with fibrosis suggesting interstitial lung disease was dominant. In the second case, inflammation with necrosis was found, but the lesions were not specific and the results were not appropriately estimated. The revision of the specimens barely resulted in the suspicion of GPA although other diseases, such as SLE and BCG, were also considered. Our cases confirmed that the microscopic pattern in GPA does not always contain evident features of vasculitis or granulomatosis with microabscesses and geographical necrosis as expected which makes it difficult to establish the diagnosis and extends the diagnostic time. Here, it is important to underline that revision of these specimens by experts in lung pathology made the diagnosis more probable.

ANCA have been recognized as an important diagnostic marker in patients with AAV. The current guidelines underlined that a positive PR3- and/or MPO-ANCA result only contributes to the diagnostic work-up for AAV but is not diagnostic. As well, negative ANCA tests cannot be the basis for AAV exclusion [22]. It has been reported that about $10-20 \%$ of GPA patients can be ANCA-negative and that they usually present with localized disease especially limited to the upper and lower respiratory tracts [23]. However, it cannot be excluded that ANCA-negativity is the result of an imperfect diagnostic test: results of some studies indicated that performing detection tests with more different ELISA kits might improve the positivity of ANCA in AAV patients even from $41.4 \%$ to $69.0 \%$ [24]. On the other hand, ANCA are not specific markers for AAV and they have been reported in a huge variety of inflammatory conditions such as connective tissue diseases, infective diseases, malignancies, and in adverse drug reactions [7]. In SLE, for example, where there is a plethora of antibodies, ANCA frequency can be up to $25 \%$ [7]. In our patients, the chimericity of ANCA was excellently demonstrated. During the multiple year duration of the disease, ANCA status was variable and an increase in ANCA concentration usually was not great which hindered the interpretation of their results. Additionally, in the first patient, anti-dsDNA were found despite the absence of SLE symptoms, causing some confusion. This case confirmed that serological tests should be performed only in the context of the clinical presentation.

As already mentioned, the diagnosis of GPA can be difficult because its symptoms are varied and can mimic other diseases. Systemic features, if they occur, are not characteristic and may last from weeks to months before evidence of specific organ involvement. In a small study by Gomez-Gomez et al. [16], the average duration of symptoms to determine the diagnosis was 5.3 months, while in a study by Kumar et al. [18], median disease duration at diagnosis was 20 months. Interesting data was presented by Yacyshyn et al. [25]: they presented the results of an anonymous survey in which respondents self-reported a diagnosis of GPA. In the patients' opinion, $36.4 \%$ of GPA cases had a delay in diagnosis of more than 1 year, and $12.3 \%$ reported a delay for more than 3 years. In our patients, disease duration at diagnosis was 4 and 6 years respectably after initial presentation despite diagnostic thoracotomy having been completed twice. The diagnosis was difficult because none of the diagnostic compartments were characteristic for GPA: there were neither clinical symptoms, radiological lesions, nor histologic patterns. In the elderly, the typical ENT symptoms are less serious. The changing pulmonary lesion character and intensity during several years of observation which was described in our patients is a rather rare phenomenon. Another element that makes recognition difficult was the nonspecific and incomplete histological pattern. However, both meticulous monitoring and thorough histological revision allowed us to determine the appropriate diagnosis and to administrate effective treatment.

In summary, our two patients represent how GPA is a great diagnostic challenge, especially in the elderly. They excellently showed the diagnostic difficulties of isolated pulmonary nodules 
and/or cavitations which usually needed invasive procedures to rule out infection or metastatic cancer. The approach requires a critical analysis of clinical and radiographical features, biomarkers, cultures and histological examinations via lung biopsy even by thoracotomy at the beginning and not only on follow up. An analysis of the presented cases leads to several conclusions. Firstly, the histological examination is not sufficient enough to establish a GPA diagnosis on its own, especially when the microscopic picture does not show evident features of vasculitis or granulomatosis with microabscesses. Secondly, the histological diagnosis of GPA is challenging and requires a lot of experience from the pathologist. Thirdly, solitary pulmonary nodules, after exclusion of neoplastic characteristics, require precise observation with regards to the development of GPA. And finally, the cases show that both experience and close cooperation between the clinicians and the pathologists are necessary in order to determine the appropriate diagnosis.

\section{Conflict of interest}

None declared.

\section{References:}

1. Jennette JC, Falk RJ, Bacon PA, et al. 2012 revised International Chapel Hill Consensus Conference Nomenclature of Vasculitides. Arthritis Rheum. 2013; 65(1): 1-11, doi: 10.1002/ art.37715, indexed in Pubmed: 23045170.

2. Watts RA, Lane SE, Bentham G, et al. Epidemiology of systemic vasculitis: a ten-year study in the United Kingdom. Arthritis Rheum. 2000; 43(2): 414-419, doi: 10.1002/1529-0131(200002)43:2<414::AID-ANR23>3.0. CO;2-0, indexed in Pubmed: 10693883.

3. Ponte C, Águeda AF, Luqmani RA. Clinical features and structured clinical evaluation of vasculitis. Best Pract Res Clin Rheumatol. 2018; 32(1): 31-51, doi: 10.1016/j.berh.2018.10.001, indexed in Pubmed: 30526897.

4. Greco A, Marinelli C, Fusconi M, et al. Clinic manifestations in granulomatosis with polyangiitis. Int J Immunopathol Pharmacol. 2016; 29(2): 151-159, doi: 10.1177/0394632015617063, indexed in Pubmed: 26684637.

5. Suwanchote S, Rachayon M, Rodsaward P, et al. Anti-neutrophil cytoplasmic antibodies and their clinical significance. Clin Rheumatol. 2018; 37(4): 875-884, doi: 10.1007/s10067018-4062-x, indexed in Pubmed: 29525845.

6. Lapraik C, Watts R, Bacon P, et al. BSR and BHPR guidelines for the management of adults with ANCA associated vasculitis. Rheumatology (Oxford). 2007; 46(10): 1615-1616, doi: 10.1093/rheumatology/kem146a, indexed in Pubmed: 17804455.

7. Weiner M, Segelmark M. The clinical presentation and therapy of diseases related to anti-neutrophil cytoplasmic antibodies (ANCA). Autoimm Rev. 2016; 15(10): 978-982, doi: 10.1016/j. autrev.2016.07.016.

8. Comarmond C, Cacoub P. Granulomatosis with polyangiitis (Wegener): clinical aspects and treatment. Autoimmun Rev. 2014; 13(11): 1121-1125, doi: 10.1016/j.autrev.2014.08.017, indexed in Pubmed: 25149391.
9. Thickett DR, Richter AG, Nathani N, et al. Pulmonary manifestations of anti-neutrophil cytoplasmic antibody (ANCA)-positive vasculitis. Rheumatology (Oxford). 2006; 45(3): 261-268, doi 10.1093/rheumatology/kei217, indexed in Pubmed: 16418204.

10. Gafoor K, Patel S, Girvin F, et al. Cavitary lung diseases: a clinical-radiologic algorithmic approach. Chest. 2018; 153(6): 1443-1465, doi: 10.1016/j.chest.2018.02.026, indexed in Pubmed: 29518379

11. Harper L, Savage CO. ANCA-associated renal vasculitis at the end of the twentieth century--a disease of older patients. Rheumatology (Oxford). 2005; 44(4): 495-501, doi: 10.1093/ rheumatology/keh522, indexed in Pubmed: 15613403.

12. Krafcik SS, Covin RB, Lynch JP, et al. Wegener's granulomatosis in the elderly. Chest. 1996; 109(2): 430-437, doi: 10.1378/ chest.109.2.430, indexed in Pubmed: 8620718.

13. Hoganson DD, From AM, Michet CJ. ANCA vasculitis in the elderly. J Clin Rheumatol. 2008; 14(2): 78-81, doi: 10.1097/ RHU.0b013e31816b2fbd, indexed in Pubmed: 18391675.

14. Chen M, Yu F, Zhang Y, et al. Antineutrophil cytoplasmic autoantibody-associated vasculitis in older patients. Medicine (Baltimore). 2008; 87(4): 203-209, doi: 10.1097/ MD.0b013e31817c744b, indexed in Pubmed: 18626303.

15. Jefferson JA. Treating elderly patients with ANCA-associated vasculitis. Clin J Am Soc Nephrol. 2015; 10(7): 1110-1113, doi: 10.2215/CJN.05350515, indexed in Pubmed: 26100458.

16. Gómez-Gómez A, Martínez-Martínez MU, Cuevas-Orta E, et al. Pulmonary manifestations of granulomatosis with polyangiitis. Reumatol Clin. 2014; 10(5): 288-293, doi: 10.1016/j. reuma.2013.12.010, indexed in Pubmed: 24529938.

17. Lin YL, Hsu YH. Wegener's granulomatosis simulates pulmonary adenocarcinoma. Tzu Chi Medical Journal. 2015; 27(1): 38-40, doi: 10.1016/j.tcmj.2013.12.001.

18. Kumar A, Dembla G, Abrol A, et al. Clinical profile and longterm outcome of granulomatosis with polyangiitis (GPA): A corporate hospital-based study from northern India. Indian Journal of Rheumatology. 2015; 10(4): 183-188, doi: 10.1016/j. injr.2015.06.001.

19. Mark EJ, Matsubara O, Tan-Liu NS, et al. The pulmonary biopsy in the early diagnosis of Wegener's (pathergic) granulomatosis: a study based on 35 open lung biopsies. Hum Pathol. 1988; 19(9): 1065-1071, doi: 10.1016/s0046-8177(88)80088-1, indexed in Pubmed: 3417290.

20. Travis WD, Hoffman GS, Leavitt RY, et al. Surgical pathology of the lung in Wegener's granulomatosis. Review of 87 open lung biopsies from 67 patients. Am J Surg Pathol. 1991; 15(4): 315-333, doi: 10.1097/00000478-199104000-00001, indexed in Pubmed: 2006712.

21. Wegener F. Wegener's granulomatosis. Thoughts and observations of a pathologist. Eur Arch Otorhinolaryngol. 1990; 247(3): 133-142, doi: 10.1007/bf00175962, indexed in Pubmed: 2190624.

22. Bossuyt X, Tervaert JWC, Arimura Y, et al. Revised 2017 international consensus on testing of ANCAs in granulomatosis with polyangiitis and microscopic polyangiitis. Nat Rev Rheumatol. 2017; 13(11): 683-692, doi: 10.1038/ nrrheum.2017.140.

23. Mukhtyar C, Flossmann O, Hellmich B, et al. EULAR recommendations for conducting clinical studies and/or clinical trials in systemic vasculitis: focus on anti-neutrophil cytoplasm antibody-associated vasculitis. Ann Rheum Dis. 2007; 66(5): 605-617, doi: 10.1136/ard.2006.062711, indexed in Pubmed: 17170053.

24. Tateyama K, Kodama S, Kishibe K, et al. A novel strategy with combined assays for detection of anti-neutrophil cytoplasmic antibody (ANCA) in clinically ANCA-negative granulomatosis with polyangiitis patients. Auris Nasus Larynx. 2017; 44(6): 735-741, doi: 10.1016/j.anl.2017.03.002, indexed in Pubmed: 28666565.

25. Yacyshyn E, Johnson A, Rode M, et al. Patient-driven online survey on the clinical manifestations and diagnostic delay of granulomatosis with polyangiitis. Joint Bone Spine. 2016; 83(5): 599-600, doi: 10.1016/j.jbspin.2015.07.014, indexed in Pubmed: 27052430. 